\title{
How safe is it to administer dual anti-HER2 block with pertuzumab and trastuzumab on a patient with life-threatening liver breast cancer metastasis?
}

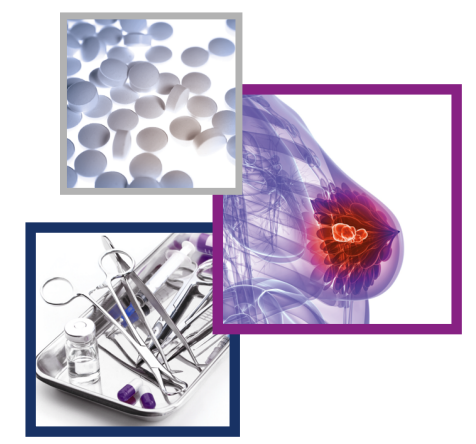

\author{
Valentina Rossi*,1 (iD), Lucia Rosalba Grillo², Fabio Di Cesare ${ }^{3}$, Enrico Saraco ${ }^{3}$, Fabrizia \\ Scardamaglia ${ }^{3}$, Francesca Svegliati ${ }^{4}$, Andrea Cortese ${ }^{5}$ \& Leonardo Vigna ${ }^{1}$ \\ ${ }^{1}$ Oncological Breast Unit, S.Camillo-Forlanini Hospital of Rome, Circ. Gianicolense 87, Rome, 00152, Italy \\ ${ }^{2}$ Patholgy Unit, S.Camillo-Forlanini Hospital of Rome, Circ. Gianicolense 87, Rome, 00152, Italy \\ ${ }^{3}$ Breast Surgery Unit, S.Camillo-Forlanini Hospital of Rome, Circ. Gianicolense 87, Rome, 00152, Italy \\ ${ }^{4}$ Radiological Breast Unit, S.Camillo-Forlanini Hospital of Rome, Circ. Gianicolense 87, Rome, 00152, Italy \\ ${ }^{5}$ Radiological Unit, S.Camillo-Forlanini Hospital of Rome, Circ. Gianicolense 87, Rome, 00152, Italy \\ *Author for correspondence: VRossi@scamilloforlanini.rm.it
}

\section{Practice points}

- HER2-positive metastatic breast cancer presenting with acute liver impairment is a rare phenomenon carry a dismal prognosis.

- Safety and effectiveness of standard anti-HER2 therapies plus chemotherapy were not assessed in patients affected by liver metastasis and hepatic failure.

- We report a case of young woman with HER2-positive metastatic breast cancer and visceral crisis who benefits from dual anti-HER2 block with pertuzumab and trastuzumab.

- At disease diagnosis, clinical examination, liver function tests and CT scan revealed diffuse liver metastasis, ascites and peritoneal carcinomatosis.

- First-line chemotherapy based on pertuzumab-trastuzumab every 3 weeks associated with weekly paclitaxel at reduced dose due to liver dysfunction was administered.

- Clinical condition and liver function tests improved in less than 2 months.

- Dual anti-HER2 block and chemotherapy improved survival by 12 months also in visceral crisis condition.

- Early initiation of standard HER2 target therapies outweigh the risks of toxicities and can be successfully used also in patients with life-threatening liver breast cancer metastasis.

Aim: We report a case of a young woman who presented with HER2 + metastatic breast cancer and visceral crisis to asses safety and effectiveness of dual anti-HER2 plus chemotherapy. Patients \& methods/materials \& methods: At the time of advanced breast cancer diagnosis, the patient presented in scanty clinical condition with severe liver function tests elevation. First-line chemotherapy based on pertuzumab-trastuzumab plus weekly paclitaxel at $50 \%$ reduced dose due to liver dysfunction was administered. Results: Improvement and normalization of liver function tests and patient's clinical condition was recorded in less than 2 months. Computed tomography revealed a partial remission of liver metastasis. Conclusions: Early initiation of standard HER2 target therapies outweigh the risks of toxicities and can be successfully used also in patients with life-threatening liver breast cancer metastasis.

First draft submitted: 6 April 2020; Accepted for publication: 15 July 2020; Published online:

4 September 2020

Keywords: dual anti-HER2 block • HER2 positive $\bullet$ metastatic breast cancer $\bullet$ visceral crisis

The overexpression of HER2 is found in 25\% of all breast cancer and results in a more aggressive disease [1]. Over recent years, a significant improvement in overall survival (OS) was reported with the approval of pertuzumab and trastuzumab in the first-line setting in addition to the antibody-drug conjugate TDM-1 and dual tyrosine kinase inhibitor lapatinib in second- and further line settings [2]. More specifically, pertuzumab was approved in June 2012 as first-line therapy in HER2 positive $(+)$ metastatic breast cancer (MBC) in combination with docetaxel

Future Medicine 
Table 1. Liver dysfunction at the time of multiple liver metastasis onset.

\begin{tabular}{|lllllll|}
\hline Date & AST U/I (5-40) & ALT U/I (5-40) & GGT U/I (<38) & LDH U/I (210-400) & $\begin{array}{l}\text { Total bilirubin mg/dl } \\
(\mathbf{0 . 2}-\mathbf{1 . 0})\end{array}$ & $\begin{array}{l}\text { Direct bilurubin mg/dl } \\
\text { (<0.2) }\end{array}$ \\
\hline 15 May 2017 & 339 & 87 & 588 & 1.444 & 6.40 & 4.80 \\
\hline 17 May 2017 & 353 & 122 & - & 1.137 & 8.20 & 6.30 \\
\hline 18 May 2017 & 351 & 144 & 929 & 1.140 & 8.80 & 6.90 \\
\hline 23 May 2017 & 200 & 133 & 1.364 & 1.343 & 11.50 & 8.50 \\
\hline 25 May 2017 & 173 & 128 & 1.595 & 1.244 & 12.70 & 9.40 \\
\hline 26 May 2017 & 166 & 128 & 1.680 & 1.247 & 12.80 & 9.50 \\
\hline
\end{tabular}

ALT: Alanine aminotransferase; AST: Aspartate aminotransferase; GGT: $\gamma$-glutamyl transpeptidase; LDH: Lactate dehydrogenase.

and trastuzumab thanks to a progression-free survival improvement of 18.7 months compared with the former trastuzumab plus docetaxel standard treatment. The prespecified final OS analysis with a 50-month follow-up also resulted in an exceptionally long median OS of 56 months in the pertuzumab arm. The most commonly reported toxicities associated with pertuzumab following discontinuation of docetaxel within the Cleopatra study were diarrhea, rash, pruritus and dry skin [2].

Safety and effectiveness provided by dual anti-HER2 block with pertuzumab and trastuzumab were not assessed in patients affected by liver metastasis and hepatic failure.

Here, we report a case with HER2 + MBC and visceral crisis where urgent initiation of anti-HER2 therapies plus chemotherapy resulted in a rapid clinical improvement and survival benefit.

\section{Case report}

In 2014, a 38-year-old female requested a medical consultation regarding a left breast mass. Mammography showed an irregular nodule with a diameter of $3.0 \mathrm{~cm}$ in the upper internal quadrant of her left breast, which was associated with ipsilateral axillary nodes enlargement. Both nodule and axillary node diagnostic biopsies by tru-cut revealed histopathological evidence of an infiltrating ductal carcinoma, grading 3, with local neuroendocrine aspects (5\%) and lymphovascular invasion. Biological characteristics of the tumor were: ER 99\%, PgR 90\%, Ki67 60\% and HER2 3+.

A subsequent computed tomography (CT) excluded distant metastasis. We adopted neoadjuvant chemotherapy as initial management of this locally advanced stage III breast cancer. Specifically, we administered four cycles of epirubicin $75 \mathrm{mg} / \mathrm{m}^{2}$ plus cyclophosphamide $600 \mathrm{mg} / \mathrm{m}^{2}$ day $1 / 21$, followed by four cycles of docetaxel $100 \mathrm{mg} / \mathrm{m}^{2}$ day $1 / 21$ plus trastuzumab $2 \mathrm{mg} / \mathrm{kg}$ (loading dose $4 \mathrm{mg} / \mathrm{kg}$ ). After completing neoadjuvant chemotherapy, there was a radiographic partial response of the tumor which was followed by a modified radical mastectomy with complete axillary dissection on 16 January 2015.

A definitive histopathological examination revealed a residual disease both in the breast and in the axillary nodes. Final stage of the disease was ypT1c (15 mm) ypN1 (1/12), ER 90\% PgR 80\% Ki67 35\% HER2 3+. We executed postoperative radiation treatment to chest wall and regional nodes and administered adjuvant trastuzumab for 1 year. Adjuvant hormonal therapy with tamoxifene $20 \mathrm{mg} /$ day plus ovarian suppression started on 17 February 2015.

The patient got back to her usual life and refused subsequent clinical and radiological monitoring. However, after onset of abdominal pain she was urgently admitted at our hospital on 12 May 2017. At that time, the clinical condition of our patient was very debilitating with extremely poor prognosis. The physical examination on admission revealed yellow skin and sclera colors, while liver palpation revealed swelling until the umbilical transverse line.

Table 1 shows the initial laboratory studies. Liver function tests showed severe elevation of aspartate aminotransferase, alanine aminotransferase, lactate dehydrogenase, $\gamma$-glutamyl transpeptidase and serum bilirubin. The serum calcium level rose up to $12.8 \mathrm{mg} / \mathrm{dl}$. Tumor markers including CA 15-3 and CEA were significantly elevated.

CT scan revealed diffuse liver metastasis, in addition to the ascites and peritoneal carcinomatosis. The tumor comprised more than $50 \%$ of the hepatic volume and the ultrasonography evaluation excluded biliary obstructive disorders which required a biliary stent. (Figure 1A) A bone scan revealed also diffuse lytic lesions in her vertebral column, pelvis and right humerus. 
(A)

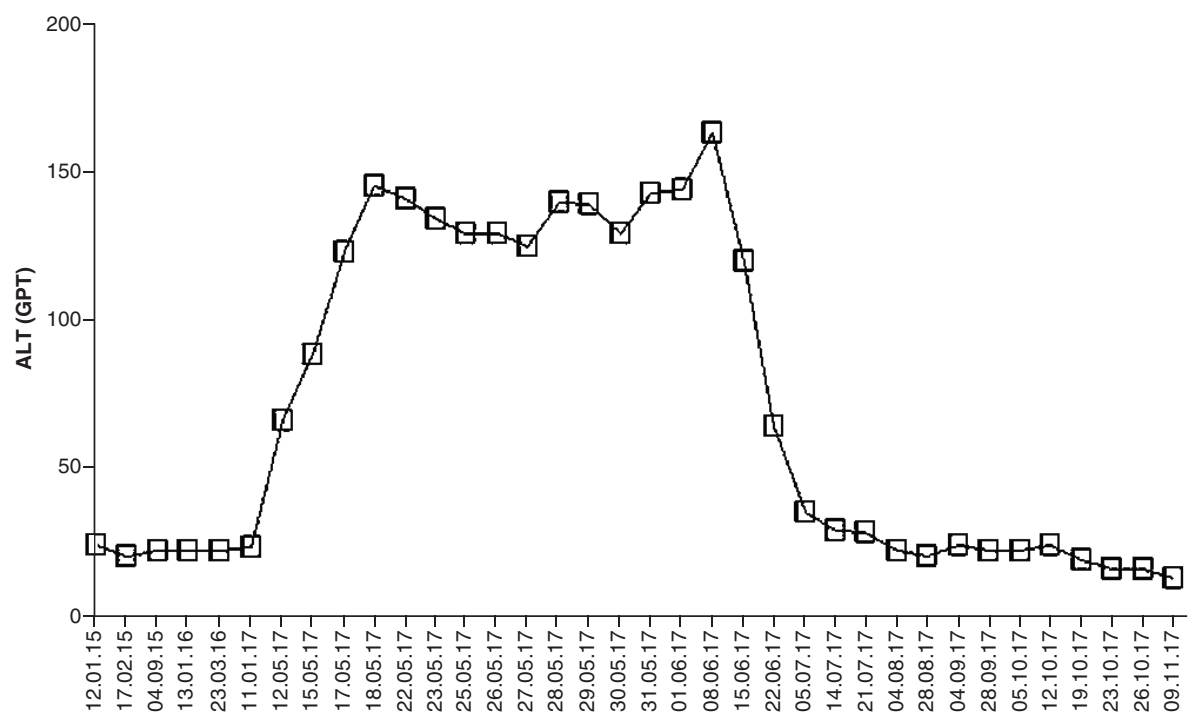

(B)

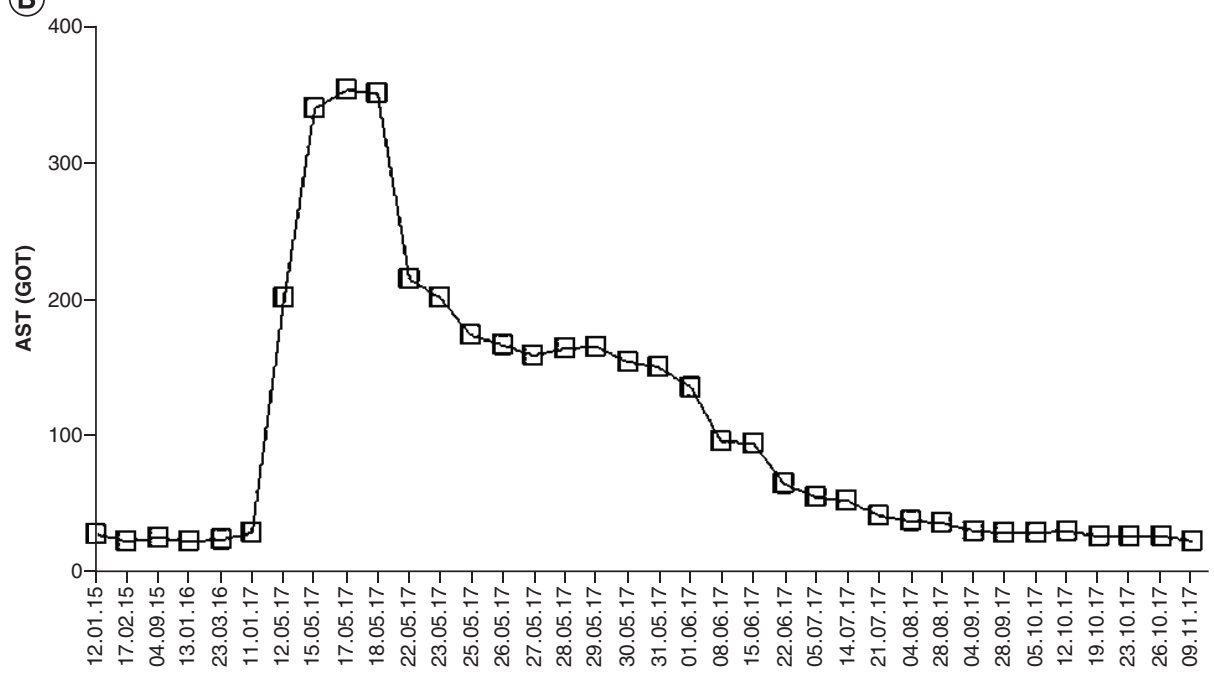

Figure 1. Liver function test modifications during pertuzumab-trastuzumab-paclitaxel therapy. (A) Alanine AminoTransferase function test curve at the time of pertuzumab-trastuzumab-paclitaxel treatment. (B) Aspartate AminoTransferase function test curve during pertuzumab-trastuzumab-paclitaxel treatment. (C) Total Bilirubin function test curve during pertuzumab-trastuzumab-paclitaxel treatment. (D) Direct Bilirubin function test curve during pertuzumab-trastuzumab-paclitaxel treatment.

ALT: Alanine aminotransferase; AST: Aspartate aminotransferase.

Considering her young age, we opted for a first-line chemotherapy cycle with pertuzumab (Total dose (DT) $840 \mathrm{mg}$ )-trastuzumab (DT:640 mg) every 3 weeks associated with weekly paclitaxel (DT:76 mg; paclitaxel 50\% reduced dose due to liver dysfunction) on 19 May 2017. This was in spite of not having any data to support the use of double anti-HER2 block in a patient with HER2+ MBC and severe liver impairment.

Due to the worsening of the hepatic impairment, subsequent paclitaxel dose was omitted on day 8 and 15 of the first cycle and reinstated on day 1 of the subsequent cycle when her liver function tests finally stopped increasing.

As shown in Figure 1, we witnessed an improvement and normalization of liver function tests in less than 2 months, which was reflected by a rapid improvement of the patient's clinical condition which moved immediately from ECOG 3 to ECOG 1.

A CT scan performed after 7 weeks of treatment also revealed a complete remission of peritoneal carcinomatosis and ascites in addition to the partial remission of liver metastasis (Figure 2). On day 8 of the third cycle the dose of paclitaxel was increased to full dosage of $80 \mathrm{mg} / \mathrm{m}^{2}$ (DT $140 \mathrm{mg}$ ). 


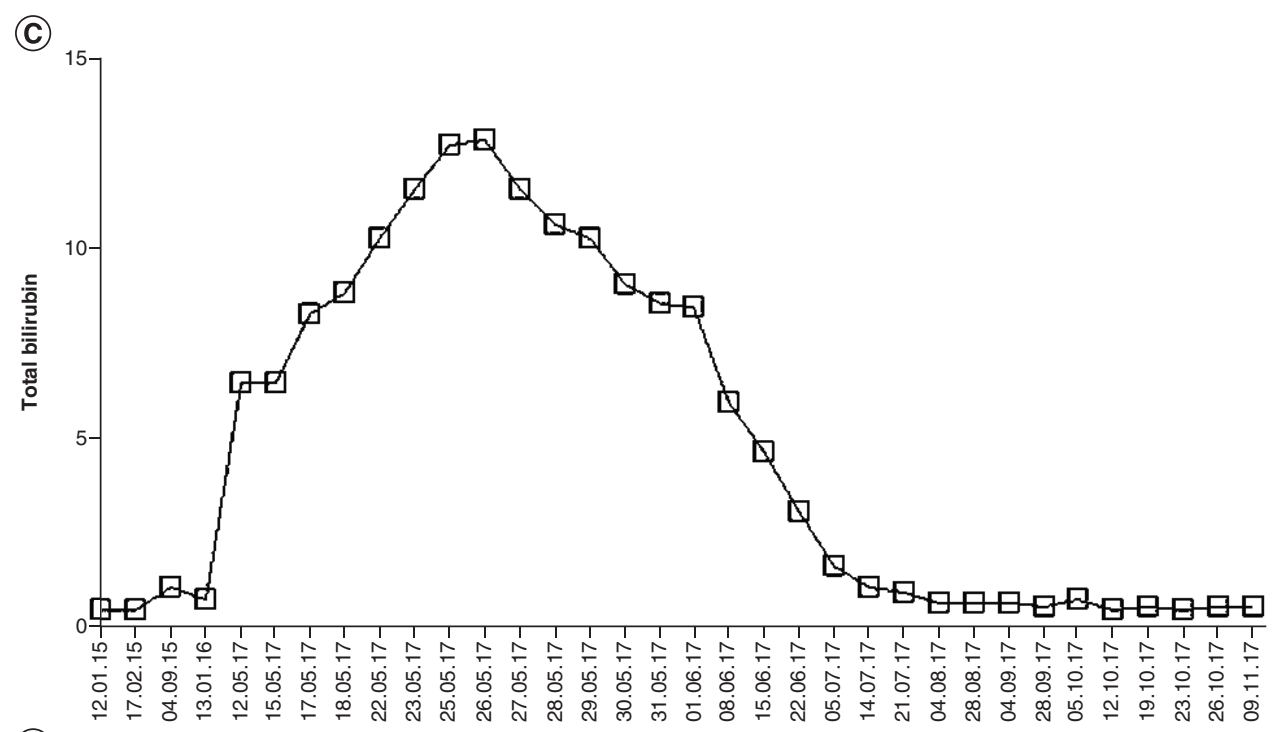

(D)

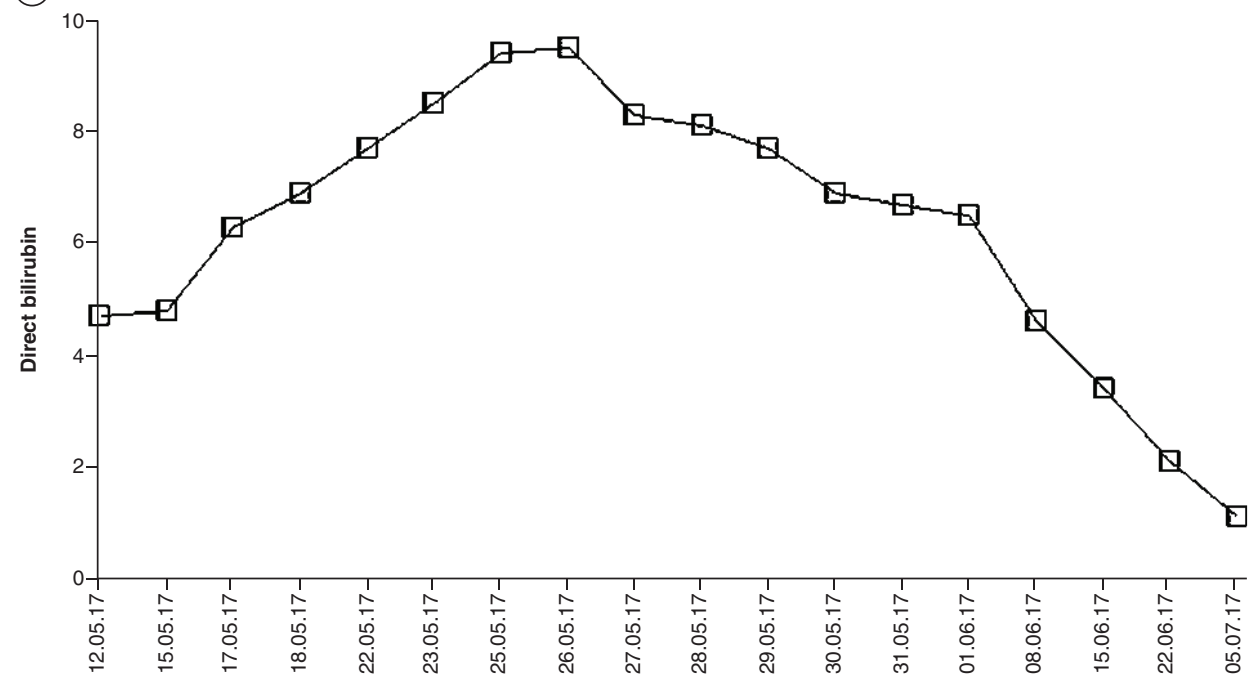

Figure 1. Liver function test modifications during pertuzumab-trastuzumab-paclitaxel therapy (cont.). (A) Alanine AminoTransferase function test curve at the time of pertuzumab-trastuzumab-paclitaxel treatment. (B) Aspartate AminoTransferase function test curve during pertuzumab-trastuzumab-paclitaxel treatment. (C) Total Bilirubin function test curve during pertuzumab-trastuzumab-paclitaxel treatment. (D) Direct Bilirubin function test curve during pertuzumab-trastuzumab-paclitaxel treatment.

ALT: Alanine aminotransferase; AST: Aspartate aminotransferase.

Due to grade 3 peripheral neurotoxicity occurrence after completing eight cycles of chemotherapy, we decided to stop paclitaxel treatment, while dual anti-HER2 block with pertuzumab and trastuzumab continued to be administered every 3 weeks until disease progression.

The peripheral neurotoxicity had moved from G3 to G1 in 2 months and the patient gained some months in good clinical conditions with persistence of neurotoxicity at G1 level in the next 6 months.

On 25 June 2018, a new CT scan revealed a disease progression with diffuse liver involvement and impairment. A subsequent rescue attempt with Kadcyla $3.6 \mathrm{mg} / \mathrm{kg}$ D1/21 was offered, but the rapid deterioration of the patient's clinical conditions and liver function tests had led to reliance on home palliative care. The patient died for hepatic encephalopathy on December 2018.

\section{Discussion}

Acute hepatic failure caused by solid tumor metastasis is a rare condition, accounting for only $0.44 \%$ of all cases of acute hepatic failure. Despite a low incidence, it carries a mortality rate of $90 \%$ also in the case of a specific 

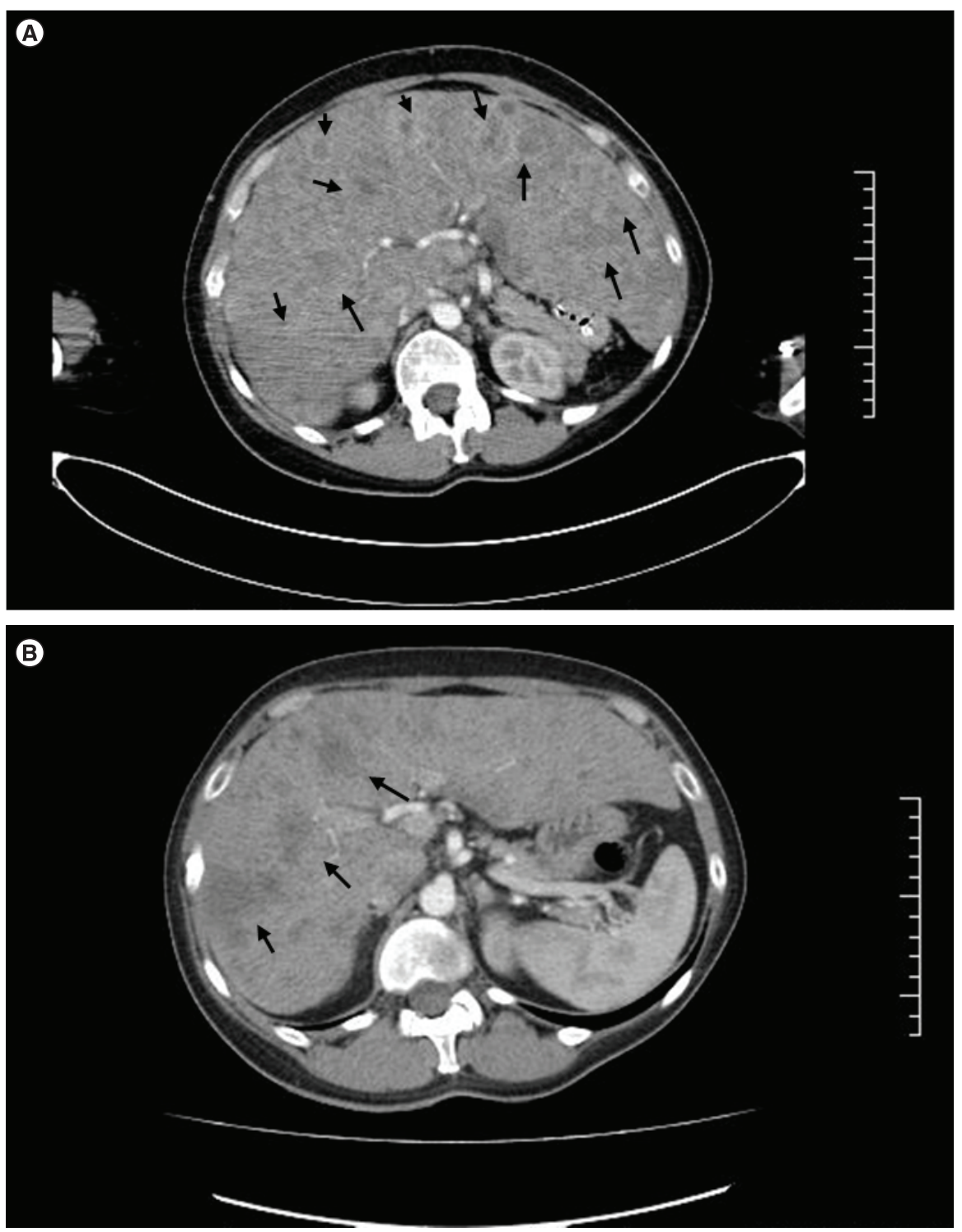

Figure 2. Liver metastasis imaging and its modifications during the treatment. (A) Liver CT scan before pertuzumab-trastuzumab-paclitaxel therapy showing multiple widespread liver metastases with significant increase in organ volume. (B) CT scan after seven weeks of pertuzumab-trastuzumab-paclitaxel therapy showing reduction in number and diameter of the multiple liver lesions with a significant reduction of organ volume.

treatment [3]. Due to such dismal prognosis early treatment with agents assuring high response rate and tolerable safety profile is the primary goal.

Here, we described our experience in the use of pertuzumab and trastuzumab in a patient with acute hepatic dysfunction and MBC. Our case proved that in HER2-addicted MBC and severe liver dysfunction, urgent initiation of therapy with HER2 target therapies and chemotherapy can reverse the severe clinical course of such condition. Our patients with acute liver failure was, indeed, successfully treated by combining paclitaxel with dual anti-HER2 block. Our decision to use weekly paclitaxel instead of docetaxel was influenced by the higher quality of its overall toxicity profile [4]. As the main excretion of paclitaxel is by hepatic pathway, the pivotal trial registered grade 1 or 2 liver toxicity in more than $50 \%$ of patients receiving paclitaxel-pertuzumab-trastuzumab. We witnessed an initial deterioration of liver functions which forced us to interrupt the administration of paclitaxel during the first cycle of treatment. The dual anti-HER2 block on its own proved to be highly and rapidly effective by interrupting 
the constant increase of liver enzymes starting from as early as the second cycle. This improvement allowed us to resume the adoption of an escalation therapy approach.

To the best our knowledge, the use of paclitaxel along with pertuzumab and trastuzumab was not well documented in terms of clinical liver impairment condition [4]. Review of the literature highlighted additional case reports in which patients with $\mathrm{MBC}$ and severe liver dysfunction were treated using single-agent chemotherapy with trastuzumab, and just in a single case report dual anti-HER2 block along with platinum chemotherapy were successfully used in the same organ dysfunction [5,6]. An additional single case report showed TDM1 benefit in the fifth-line setting for HER2-positive MBC and liver dysfunction [7].

To our knowledge, there are no other reports with severe hepatic dysfunction treated successfully with dual anti-Her2 block along with taxane based chemotherapy in first-line setting, according to the current standard approach. One case study used docetaxel in a newly diagnosed patient with breast cancer and hepatic visceral crisis, but unlike our patient who had a total peak of $12.80 \mathrm{mg} / \mathrm{dl}$, this patient had a peak bilirubin of $2.2 \mathrm{mg} / \mathrm{dl}$ [8]. In our literature review, we found two other Japanese case studies on HER2+ MBC and severe liver dysfunction, with one of the cases using trastuzumab monotherapy and the other using trastuzumab and transitioning from paclitaxel to vinorelbine when severe liver dysfunction developed [5]. Finally, we found a single case of a patient with MBC and severe liver dysfunction treated with dual anti-HER2 block. The initial plan for this patient was to start the standard first-line treatment with pertuzumab, trastuzumab and docetaxel, but the choice fell on platinum-based chemotherapy due to the severe liver dysfunction [6].

\section{Conclusion}

Our experience suggests that standard dual anti-HER2 block along with taxane-based chemotherapy should be adopted in case of patients with MBC and liver dysfunction in order to reverse a potentially fatal outcome. We believe that in patients with severe liver dysfunction the benefits of HER2 target therapies outweigh the risks and should not be withheld from these critical patients.

\section{Author contributions}

All the authors contributed to the literature, review, intellectual content and drafting of this paper.

\section{Financial \& competing interests disclosure}

The authors have no relevant affiliations or financial involvement with any organization or entity with a financial interest in or financial conflict with the subject matter or materials discussed in the manuscript. This includes employment, consultancies, honoraria, stock ownership or options, expert testimony, grants or patents received or pending, or royalties.

No writing assistance was utilized in the production of this manuscript.

Ethical conduct of research

The authors state that they have obtained appropriate institutional review board approval or have followed the principles outlined in the Declaration of Helsinki for all human or animal experimental investigations. In addition, for investigations involving human subjects, informed consent has been obtained from the participants involved.

\section{Open access}

This work is licensed under the Attribution-NonCommercial-NoDerivatives 4.0 Unported License. To view a copy of this license, visit http://creativecommons.org/licenses/by-nc-nd/4.0/

\section{References}

Papers of special note have been highlighted as: $\bullet$ of interest; $\bullet \bullet$ of considerable interest

1. Sorlie T, Perou CM, Tibshirani R et al. Gene expression patterns of breast carcinomas distinguish tumor subclasses with clinical implications. Proc. Natl Acad. Sci. USA 98(19), 10869-10874 (2001).

2. Swain SM, Baselga J, Kim SB et al. Pertuzumab, trastuzumab, and docetaxel in HER2-positive metastatic breast cancer. N. Engl. J. Med. 372(8), 724-734 (2015).

-• A randomized study where the role of dual anti-HER2 block was assessed in a large number of patients.

3. Nazario HE, Lepe R, Trotter JF. Metastatic breast cancer presenting as acute liver failure. Gastroenterol. Hepatol. 7(1), 65-66 (2011).

4. Bachelot T, Ciruelos E, Schneeweiss A et al. Preliminary safety and efficacy of first-line pertuzumab combined with trastuzumab and taxane therapy for HER2-positive locally recurrent or metastatic breast cancer (PERUSE). Ann. Oncol. 30(5), 766-773 (2019). 
-• A randomized study where the role of dual anti-HER2 block was assessed in a large number of patients.

5. Ota Y, Ishikawa T, Yamada A et al. [Long-term survival of a breast cancer patient with liver metastasis treated with trastuzumab and Paclitaxel]. Gan To Kagaku Ryoho 37(6), 1091-1094 (2010).

- A clinical experience where anti-HER2 therapies were tested in patients with acute liver impairment.

6. Macias MN, Shin DS, Ledezma B, Sadeghi S. Metastatic human epidermal growth factor 2 (HER2/neu) amplified breast cancer with acute fulminant hepatitis responding to trastuzumab, pertuzumab and carboplatin. BMJ Case Rep. 2014, bcr2013203400 (2014).

- A clinical experience where anti-HER2 therapies were tested in patients with acute liver impairment.

7. Sharp A, Johnston SRD. Dose-reduced trastuzumab emtansine: active and safe in acute hepatic dysfunction. Case Rep. Oncol. 8(1), 113-121 (2015).

8. Goswami R, Babich M, Farah KF. Occult breast malignancy masquerading as acute hepatic failure. Gastroenterol. Hepatol. 7(1), 62-65 (2011). 
